\title{
NanoScale Quantitative Mechanical Property Mapping Using Peak Force Tapping Atomic Force Microscopy
}

\author{
S. C. Minne, Y. Hu, S. Hu, B. Pittenger, C. Su \\ Veeco Instruments, 112 Robin Hill Road, Santa Barbara, CA 93110
}

The AFM has long been recognized for its ability to either image surfaces at the nanoscale, or to determine mechanical properties at the nanoscale. However, until recently, the combination of these two capabilities was often a compromise between achievable imaging rate, and amount or quality of material property data collected - Examples are Phase Imaging, force volume maps collected over hours, or AFM images with a few select measurement points for mechanical properties. Here, we present recent advances in AFM technology that allow the collection of mechanical data (modulus, adhesion, deformation, dissipation) at normal AFM imaging rates. We also present an implementation of this technology that greatly simplifies AFM tapping operation while at the same time making it more robust, and the interaction more controlled.

This talk will present a new way to control atomic force microscope and acquire quantitative mechanical properties at the nanometer scale. In this new technology, the instantaneous interaction force during tapping is used to control tip-surface interaction while imaging. The force control accuracy allows imaging at $10 \mathrm{pN}$ interaction force without drift. The accurate low force control enables high resolution imaging in ambient and fluid. More importantly, the measurement records full interaction force curve and calculate modulus, deformation (hardness), adhesion and energy dissipation concurrent with the topographic imaging process.

The control method, which we call Peak Force Tapping (PFT), was used to study OmpG membrane pore at neutral $\mathrm{pH}$ buffer to locate the opening core (Figure 1). When higher interaction force is used, from hundreds $\mathrm{pN}$ to tens of $\mathrm{nN}$ peak force, a broad range of material property can be studied quantitatively. The data of homogeneous benchmark samples ranging from 0.6GPa to $100 \mathrm{GPa}$ demonstrated consistency of the AFM nanomechanical property with other mechanical measurements.

In another case study, we compared peak force tapping control with conventional tapping mode. In contrast to tapping mode, which responds to both short range and long range interactions, the peak force tapping uses only short range interaction force for controlling feedback but record full range interaction forces. In a study of poly(n-butylacrylate) molecular brushes we have found the short range interaction control is the key to achieve molecular resolution in collapsed molecules (Figure 3). Simultaneous quantitative mechanical analysis also revealed substructure of single molecule chain. Detail mechanism of the brush molecule collapsing and substructure is still under study. The aspects regarding ease of use of the new control technology in all environments, such as ambient, fluid vacuum and varying temperatures, will be discussed and demonstrated. 

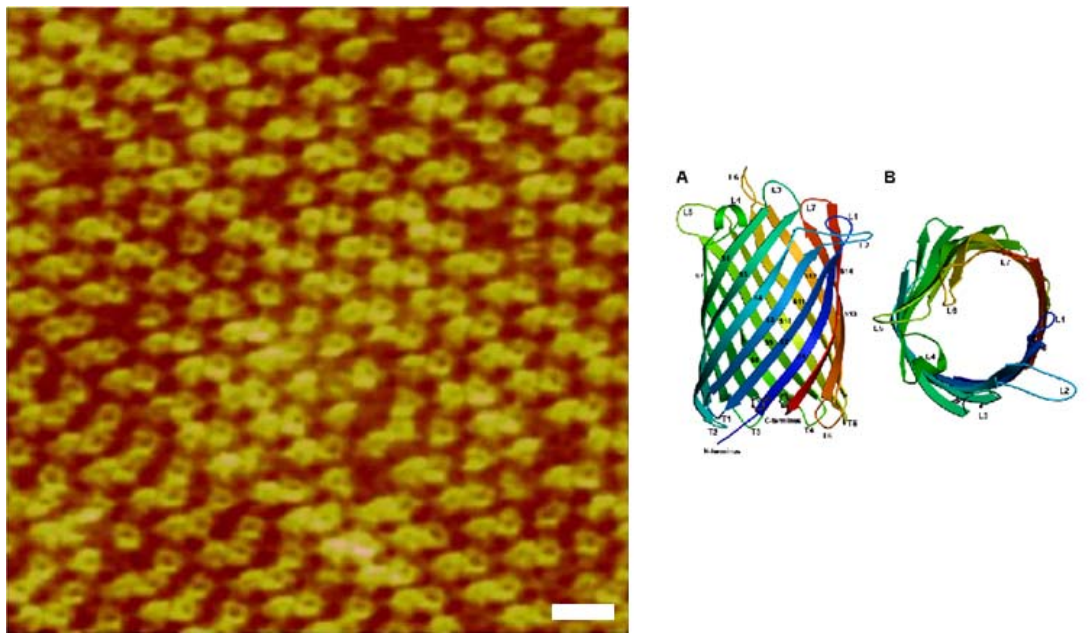

Figure 1: OmpG membrane pores imaged with a Peak Force Tapping using a peak force setpoint of $50 \mathrm{pN}(10 \mathrm{~nm}$ scale bar). Image was acquired with a MultiMode using Peak Force Tapping in a buffer solution. Inset: structure of OmpG.[ref] Courtesy C. Bippes and D. Muller, University of Technology Dresden.

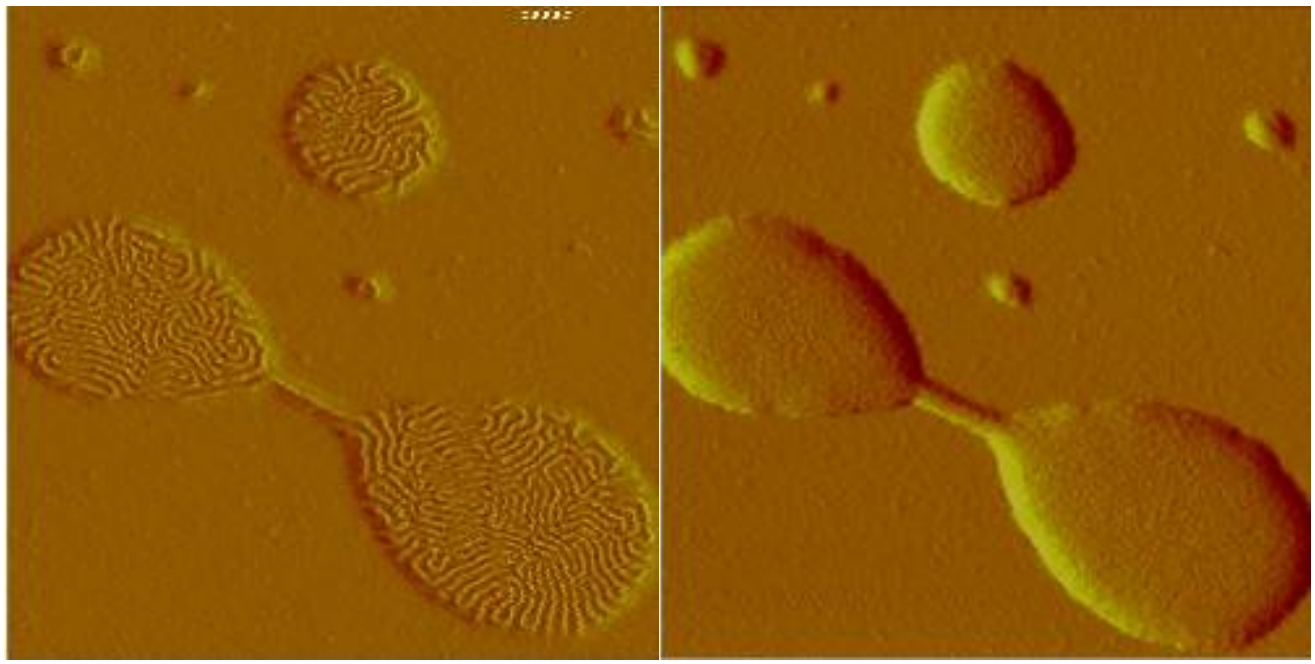

Figure 2: PBA Polymer brush sample imaged on a MultiMode 8 using (left) Peak Force Tapping, and (right) TappingMode. Energy dissipated by the polymer brush in the far field prevents tapping mode from resolving the surface. The direct force control of Peak Force Tapping clearly resolves the structure. Scan size $2 \mu \mathrm{m}$. 